\title{
Human antiretinal antibodies in toxoplasma retinochoroiditis
}

\author{
R M Whittle, G R Wallace, R A Whiston, D C Dumonde, M R Stanford
}

\begin{abstract}
Backgroundlaims-Toxoplasma retinochoroiditis (TR) is an important cause of blindness and visual morbidity, affecting young adults. It has been postulated that some of the retinal damage observed in TR is due to antiretinal autoimmune mechanisms.

Methods-Humoral antiretinal autoimmunity in TR was investigated by indirect immunofluorescence (IIF) on normal human cadaveric retina and by a human retinal S-antigen ELISA. 36 patients with TR were separated on clinical grounds into those with first recurrence of disease $(n=18)$ or those with multiple recurrences $(n=18)$. Patients were also segregated into those with active $(n=28)$ or quiescent disease $(n=8)$. Serum from 16 normal controls (six with positive toxoplasma serology and 10 without) with no evidence of eye disease and 12 patients with idiopathic retinal vasculitis (IRV) were also tested.
\end{abstract}

Results-Sera from 34 of the 36 patients (94\%) with TR demonstrated photoreceptor layer reactivity by IIF contrasting with six of 16 normal controls $(p=<0.001)$ and three of 12 IRV patients $(p=<0.001)$. Titres of antiphotoreceptor antibody were also higher among TR patients than controls. Sera from 27 of the 36 TR patients, 10 of 16 normals, and nine of 12 retinal vasculitis patients possessed anti-human retinal $S$-antigen antibodies at a titre of $1: 400$ or more as assessed by ELISA $(p=>0.05)$. Antiretinal autoantibody as detected by IIF did not run in parallel with $\mathbf{S}$-antigen reactivity.

Conclusions-The data indicate that the extent of antiretinal reactivity within $T R$ is not accounted for by anti-S-antigen antibodies alone. This remarkably high prevalence of antiphotoreceptor antibody in TR as opposed to that found in either healthy or disease controls suggest that these antibodies may be co-pathogenic in toxoplasma retinochoroiditis. (Br F Ophthalmol 1998;82:1017-1021)

Medical Eye Unit, St

Thomas's Hospital, London

M R Stanford

Correspondence to: Mr M R Stanford, Medical Eye Unit, North Wing, St Thomas's Hospital, Lambeth Palace Road, London SE1 7EH.

Accepted for publication 17 March 1998 inflammation, being cited as the aetiological agent for posterior uveitis in up to $85 \%$ of case in some surveys. ${ }^{2}$ Toxoplasma retinochoroiditis
(TR) is a cause of visual handicap, particularly in young people and is characterised by focal retinal inflammation and vitritis causing short term impairment of vision or permanent visual loss. A recent study ${ }^{3}$ estimated an incidence of $0.4 / 100000$ per year for people born in the UK rising to $57 / 100000$ per year for people born in west Africa now resident in the UK. The majority of cases of TR are thought to originate from congenital infection with disease expression at a later date. ${ }^{45}$ Ocular toxoplasmosis is the most common manifestation of congenital $T$ gondii infection. ${ }^{6}$

The pathogenesis of TR is not fully understood although a number of mechanisms of tissue damage have been postulated. These include parasite induced retinal cell lysis, damage from a soluble parasite toxin, bystander damage of retinal cells during the immune response against the parasite, hypersensitivity reactions to the parasite within the retina, and antiretinal autoimmune mechanisms. ${ }^{7}$ In parasite mediated damage, the pathology was hypothesised to have been caused by the local invasion and subsequent lysis of retinal cells and also by the release of a soluble toxin from the parasite. ${ }^{89}$ In vitro experiments have demonstrated that the parasite is able to infect all nucleated cell types and cause host-cell lysis, although direct evidence of parasite reactivation from the bradyzoite form in disease relapse is lacking. ${ }^{11}{ }^{11}$ The potential toxic effects of the organism have not been supported since subsequent studies in which culture supernatants of $T$ gondii were injected into the vitreous in a rabbit model of TR did not cause significant pathology. ${ }^{12}$ Possible immunopathological mechanisms in TR have been suggested to be mediated by "bystander damage" to uninfected retinal cells during the immune response and by hypersensitivity reactions within the retina. The bystander theory of destruction of uninfected retinal cells remains untested. Delayed type hypersensitivity reactions to toxoplasma antigens exposed in the retina are supported by the granulomatous appearance of the lesion, ${ }^{13}$ although studies using a non-human primate model indicate that hypersensitivity reactions alone are not responsible for the necrotising retinitis of recurrent toxoplasmosis. ${ }^{14}$

The proposal that autoimmunity may contribute to retinal damage in TR is supported by the detection of antiretinal immunological responses among TR patients. Lymphoproliferative responses to bovine retinal $\mathrm{S}$-antigen (b-SAg) were detected in three of five TR patients $(60 \%)$ in a study by Nussenblatt et al. ${ }^{15}$ This compared with four of the $24(16 \%)$ 
patients with other forms of uveitis. In a separate study, antiretinal responses to human retinal homogenate were found in four of four patients with active TR and six of 13 TR patients where disease had settled, and the largest proliferative responses were found in patients with active disease. ${ }^{16}$ Studies of the humoral responses to b-SAg in TR patients have demonstrated a higher prevalence and titre of anti-b-SAg antibodies in TR patients than in controls as determined by enzyme linked immunosorbent assay (ELISA) reactivity ${ }^{17}$ and antiretinal antibodies have been shown to correlate with disease activity in TR. ${ }^{18}$ Conversely, other studies have reported a background of anti-b-SAg antibodies in normal individuals as well as a failure to discriminate between non-toxoplasma uveitis patients, TR patients, and normal controls in antibody prevalence to either retinal S-antigen or interphotoreceptor retinoid binding protein (IRBP). ${ }^{19}$ It has also been noted that a proportion of sera from non-toxoplasma uveitis patients and normal controls can react with either bovine retinal $S$-antigen, human retinal S-antigen (h-SAg), or both. ${ }^{19}$

Despite current evidence of antiretinal responses in TR patients to soluble retinal proteins, controversy exists concerning their role in retinal damage during disease. Responses against insoluble membrane bound antigens have not been evaluated. We therefore examined the patterns of reactivity against human retina by indirect immunofluorescence and compared these antibody responses with those directed against human retinal S-antigen.

\section{Materials and methods}

Sera were obtained from 36 patients attending St Thomas's Hospital with clinical toxoplasma retinochoroiditis (mean age 31.6 years) defined as panuveitis in association with an area of focal retinitis adjacent to an old chorioretinal scar. The patients were subdivided in two ways. Firstly, individuals presenting with symptoms for the first time were defined as "first recurrence" (of disease) patients $(n=18)$ with those presenting with clinical symptoms subsequent to a first presentation defined as "multiple recurrence" patients $(n=18)$. Secondly, subjects were segregated by disease activity into active $(n=28)$ and quiescent groups ( $=8$ ). Blood samples were collected by venepuncture, serum separated, and stored at $-40^{\circ} \mathrm{C}$ before serological testing. All patients were seropositive for toxoplasma by the SabinFeldman dye test. Serum samples from 16 age matched (mean 34.3 years) healthy individuals with no history of eye disease were obtained as normal controls and sera from 12 age matched individuals (mean 36.2 years) with idiopathic retinal vasculitis formed an eye disease control group. Idiopathic retinal vasculitis (IRV) was diagnosed on the basis of evidence of cells in the vitreous and retinal vascular involvement and confirmed by fluorescein angiography. These sera were also tested for anti-toxoplasma antibodies.
RETINAL CRYOSTAT SECTIONS

A novel method of obtaining cryostat sections of human retina was employed utilising segments of cucumber as an inert tissue mountant in a modification of a technique used for conjunctival biopsies. ${ }^{20}$ Normal human eyes from cadavers were collected from the Bristol Eye Hospital and kept at $4{ }^{\circ} \mathrm{C}$ until preparation. Eyes were typically 24-48 hours post mortem when collected. Retinas were removed and placed in $5 \mathrm{ml}$ phosphate buffered saline (PBS) for 10 minutes. Flat segments $8-10 \mathrm{~mm}$ across were guided onto a scalpel blade and mounted on square pieces of cucumber. Retinal cucumber mounts were then placed onto a strip of filter paper, covered lightly with OCT compound $(\mathrm{BDH})$, left to stand for 2 minutes, and rapidly frozen in an isopentane bath suspended in liquid nitrogen. Retinal cucumber blocks were transferred to a $-70^{\circ} \mathrm{C}$ freezer and stored until sectioning. Blocks were oriented "side on" and sections cut transversely ( $7 \mu \mathrm{m}$ thickness) in a cryostat onto gelatine coated slides. Following air drying for 1 hour, sections were stored at $-70^{\circ} \mathrm{C}$ until use. Retinal morphology was assessed after haematoxylin and eosin staining.

\section{INDIRECT IMMUNOFLUORESCENCE ASSAY}

Volumes of $50 \mu \mathrm{l}$ of either diluted in house "positive control" sera, diluted irrelevant antibody control, diluted patient sera, and healthy control sera (range 1:10-1:160) or PBS were placed in each well and incubated for $40 \mathrm{~min}$ utes at room temperature. Sections were washed for 15 minutes in a PBS bath fitted with a magnetic stirrer. A volume of $50 \mu \mathrm{l}$ diluted (1:60 in PBS) fluorescein isothiocyanate (FITC) conjugated rabbit antisera specific for polyclonal anti-human Ig (Dako) was added to each section and slides incubated for 30 minutes in a darkened staining chamber at room temperature. Following a further PBS wash, slides were mounted, viewed under epi-illumination fluorescence, and scored blind. Staining patterns were reviewed by a second observer. Samples were ascribed an end point titre of the reciprocal of the highest serum dilution demonstrating definite reactivity. Sera lacking reactivity at the 1:10 dilution were assigned a titre of "less than 10 ". Serum was also tested on rat liver kidney blocks to detect non-organ specific autoantibodies. Antibody reactivity was defined as above.

PURIFICATION OF HUMAN S-ANTIGEN

Human S-antigen was isolated from human cadaveric eyes from which the corneas had previously been removed. Human S-antigen was purified by the use of a modified technique described by Kasp et al. ${ }^{21}$ Protein purity was assessed by SDS-PAGE with Coomassie blue staining of the gels which revealed a protein band at $50 \mathrm{kD}$. Identity was confirmed by western blotting using a mouse IgG monoclonal antibody directed against bovine S-antigen with demonstrated cross reactivity with the human homologue (kindly provided by Dr L Donoso, Wills Eye Hospital, Philadelphia, USA). 
HUMAN S-ANTIGEN ELISA

Retinal S-antigen was coated onto microtitre plate wells (Sterilin UK Ltd) at $2 \mu \mathrm{g} / \mathrm{ml}$ in PBS $\mathrm{pH} 7.4$ overnight at $4^{\circ} \mathrm{C}$. Following three washes with PBS containing $0.1 \%$ Tween 20 the wells were blocked with $150 \mu 10.4 \%$ bovine serum albumin (Sigma UK Ltd) for 30 minutes at room temperature. Plates were then washed and duplicates of $100 \mu \mathrm{l}$ diluted serum in a doubling dilution range from $1 / 25$ to $1 / 3200$ in PBS $0.1 \%$ Tween-20 plus $20 \%$ fetal calf serum (FCS) were added. Each assay plate included a dilution range of a positive control serum. After 1 hour at $37^{\circ} \mathrm{C}$ and overnight incubation at $4^{\circ} \mathrm{C}$ plates were washed and 100 $\mu \mathrm{l}$ rabbit anti-human polyclonal Ig conjugated with horseradish peroxidase (Dako UK Ltd) diluted 1:8000 in PBS $0.1 \%$ Tween-20 was added and incubated for 1 hour at $37^{\circ} \mathrm{C}$. After washing, plates were incubated with $100 \mu \mathrm{l}$ TMB substrate containing $0.2 \%$ hydrogen peroxide for 10 minutes at room temperature. The reaction was stopped with $50 \mu 1 \quad 12.5 \%$ hydrogen sulphate per well and coloured product measured at $450 \mathrm{~nm}$.

End point titres were assigned as the reciprocal of the last dilution to yield an optical density (OD) value greater than $2 \mathrm{SD}$ above the mean value of the wells lacking serum.

\section{STATISTICAL ANALYSIS}

The data generated were non-parametric and appropriate statistical analysis was performed using $2 \times 2$ contingency tables generating $\chi^{2}$ values. The variables of the $2 \times 2$ tables were of presence or absence of antiretinal antibody at a given serum dilution within the patient and control groups. A p value of less than 0.05 was considered significant.

\section{Results}

Retinal sections of appropriate quality for the performance of indirect immunofluorescence studies were obtained by the cucumber mountant method. Several retinal staining patterns were seen, including reactivity to the photoreceptor cell (Figs 1 and 2). Positive fluorescence of the photoreceptor cell cytoplasm, external limiting membrane, and the inner segments of the photoreceptor rods and cone bodies was collectively defined as "photoreceptor layer" reactivity. This study revealed a high prevalence of photoreceptor layer reactivity among TR patients with 34 of 36 TR patients (94\%) demonstrating reactivity at the $1: 10$ screening dilution contrasting with six of 16 normal con-

Table 1 Seroprevalence and titre of antiretinal antibodies in patients with toxoplasma retinochoroiditis, idiopathic retinal vasculitis, and normal controls as assessed by indirect immunofluorescence of human retina

\begin{tabular}{llll}
\hline & Group & \\
\cline { 2 - 4 } & $\begin{array}{l}\text { Toxoplasma } \\
\text { retinochoroiditis (n=36) }\end{array}$ & $\begin{array}{l}\text { Normal } \\
\text { controls; } \text { no eye } \\
\text { disease }(n=16)\end{array}$ & $\begin{array}{l}\text { Idiopathic retinal } \\
\text { vasculitis }(n=12)\end{array}$ \\
\hline $\begin{array}{llll}\text { Antiretinal antibody specificity } \\
\text { Photoreceptor layer: }\end{array}$ & $94 \%(34) \mathrm{p}=<0.001$ & $37 \%(6)$ & $25 \%(3)$ \\
$\begin{array}{c}\text { Titres of } 10 \text { or more } \\
\text { Titres of } 40 \text { or more }\end{array}$ & $58 \%(21) \mathrm{p}=<0.001$ & $6 \%(1)$ & $8 \%(1)$ \\
$\begin{array}{l}\text { Neural retina: } \\
\text { Titres of } 10 \text { or more } \\
\text { Titres of } 40 \text { or more }\end{array}$ & $31 \%(11) \mathrm{p}=>0.5$ & $31 \%(5)$ & $33 \%(4)$ \\
& $25 \%(9) \mathrm{p}=>0.5$ & $13 \%(2)$ & $8 \%(1)$ \\
\hline
\end{tabular}

Table 2 Seroprevalence and titre of antiphotoreceptor layer antibodies in patients with different disease categories of toxoplasma retinochoroiditis as assessed by IIF of human retina

\begin{tabular}{lll}
\hline & \multicolumn{2}{l}{$\begin{array}{l}\text { Antiphotoreceptor layer } \\
\text { antibody titre }\end{array}$} \\
\cline { 2 - 3 } $\begin{array}{l}\text { Toxoplasma retinochoroiditis } \\
\text { disease category }\end{array}$ & 10 & $\geqslant 40$ \\
\hline Single recurrence (18) & 17 & 11 \\
Multiple recurrence (18) & 17 & 10 \\
Active disease (28) & $\mathrm{p}=>0.5 \mathrm{NS}$ & $\mathrm{p}=>0.5 \mathrm{NS}$ \\
Quiescent (8) & 26 & 15 \\
& 8 & 6 \\
& $\mathrm{p}=0.35 \mathrm{NS}$ & $\mathrm{p}=0.29 \mathrm{NS}$ \\
\hline
\end{tabular}

trols $\left(\chi^{2}=17.34, \mathrm{p}=<0.001\right)$ and three of 12 patients with IRV $\left(\chi^{2}=24.6, \mathrm{p}=<0.001\right)$ (Table 1). At a 1:40 serum dilution the prevalence of antiphotoreceptor layer antibodies in TR patients was $58 \%(21 / 36)$ compared with only $6 \%(1 / 16)$ of normal controls $\left(\chi^{2}=12.30\right.$, $\mathrm{p}=<0.001)$ and one of 12 IRV patients $\left(\chi^{2}=\right.$ $9.06, p=0.0027)$. Twenty five per cent of TR patients had an antiphotoreceptor layer titre of 80 or more compared with none of either normal individuals or IRV patients $\left(\chi^{2}=4.87, \mathrm{p}=\right.$ $0.03)$. There was no significant difference in prevalence of photoreceptor layer antibodies between the normal controls and retinal vasculitis patients at either a $1: 10\left(\chi^{2}=0.49, \mathrm{p}=\right.$ $>0.5)$ or $1: 40$ serum dilution $\left(\chi^{2}=0.55, \mathrm{p}=\right.$ $>0.5$ ).

Neural retina reactivity (represented by perinuclear staining of inner nuclear layer cells) was found at a similar prevalence among the patient and control groups (Table 1). Nonparametric analysis revealed no significant difference in antiphotoreceptor layer antibodies between active and quiescent TR patients. Additionally, TR patients presenting for the first time exhibited similar prevalence and titre of photoreceptor layer reactivity to those with multiple disease recurrences (Table 2).

Analysis of healthy control sera (data not shown) revealed that the presence of antibodies to toxoplasma did not generate a high prevalence of characteristic antiretinal reactivity staining patterns. Thus, the hypothesis that antibodies to toxoplasma antigens are cross reactive with retinal components was not supported by the results in this study. No evidence of non-organ specific serum autoantibodies was detected in TR patients, normal controls, or IRV patients by IIF of rat liver kidney substrate (data not shown).

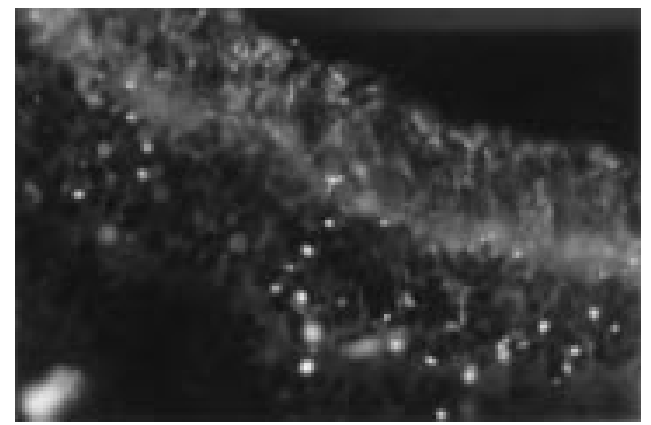

Figure 1 Indirect immunofluorescence of human retina (×40). Negative staining of all retinal layers. Note lipofuscin fragments of retinal pigment epithelia distributed across the section. 


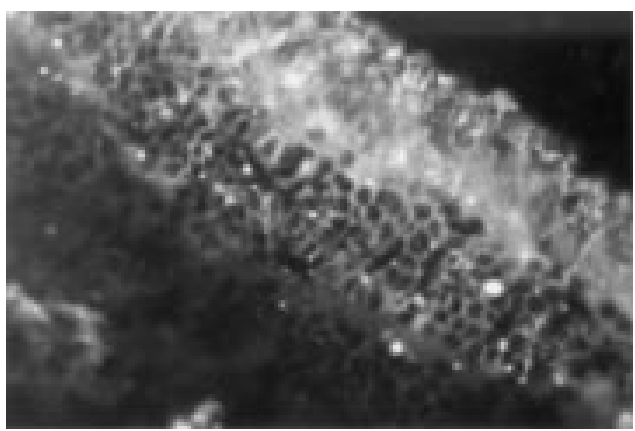

Figure 2 Indirect immunofluorescence of human retina $(\times 40)$. Positive staining of photoreceptor cell cytoplasm and in both rods and cones by antibodies present in serum from a patient with TR (serum dilution 1:40).

Table 3 Serum end point titre distribution of anti-human $S$-antigen antibodies in patients with TR, IRV, and normal healthy controls by ELISA

\begin{tabular}{lll}
\hline & $\begin{array}{l}\text { End point } \\
\text { titres } \\
>400\end{array}$ & $\begin{array}{l}\text { End point } \\
\text { titres } \\
\geqslant 3200\end{array}$ \\
\hline Toxoplasma retinochoroiditis & $27 / 36(75 \%)$ & $11 / 36(31 \%)$ \\
Idiopathic retinal vasculitis & $9 / 12(75 \%)$ & $5 / 12(42 \%)$ \\
Healthy controls & $10 / 16(63 \%)$ & $5 / 16(31 \%)$ \\
\hline
\end{tabular}

Antibody titres to human S-antigen above 400 were detected in $75 \%$ of the TR patients and IRV patients and $63 \%$ of the healthy controls ( $>0.5$ for all groups: Table 3). No particular association between antibody end point titre distribution and group tested could be established. No relation could be determined between TR disease activity, number of recurrences, and human S-antigen reactivity. Moreover, no correlation between the IIF and ELISA data was found, with some sera demonstrating high levels of S-antigen reactivity without antiphotoreceptor antibody positivity and others low S-antigen antibody titre with high levels of antiphotoreceptor antibodies.

\section{Discussion}

The aim of this study was to evaluate humoral antiretinal reactivity against human retinal tissue by indirect immunofluorescence and compare this with antibody reactivity detected by the anti-hSag ELISA. We have demonstrated a high prevalence of antiretinal antibodies which appear to be directed at the photoreceptor layer in TR patients compared with both IRV patients and normal controls. It was also apparent that a background of antibody reactivity against both the photoreceptor layer and neural retina is present in a proportion of normal individuals. TR patients have higher titre antiphotoreceptor layer antibodies than those found in normal and IRV patients. However, neither disease activity nor progression appeared to significantly influence the cellular or subcellular specificity, titre or prevalence of antiretinal antibodies in TR patients. While no differences could be determined between the level of antibody detected, the characteristics of this antibody response may vary in terms of antibody class, subclass, and affinity. Further investigations are required to examine this possibility. No significant or consistent pattern of non-organ specific autoantibodies was de- tected in the sera of either TR patients or controls indicating that there was no underlying autoantibody reactivity which may mimic or influence antiretinal staining patterns.

Human retinal S-antigen was selected for ELISA studies as this was considered more relevant than responses to the bovine homologue in light of previous findings. ${ }^{19}$ A higher background of reactivity among normal individuals is reported here than in other studies with bovine ${ }^{18}$ and human S-antigen ${ }^{19}$ although the data support previous findings that titres of antibodies to $\mathrm{S}$-antigen are not significantly different between TR patients and normal controls. Antiphotoreceptor layer reactivity was encountered among some TR patients with very low titres of anti S-antigen antibodies and photoreceptor reactivity was not consistent with retinal S-antigen reactivity. Retinal $\mathrm{S}$-antigen is found throughout the photoreceptor cell cytoplasm from the outer plexiform layer to the outer segments of the rods and cones. The ELISA results and the pattern of IIF reactivity suggest that a wider range of antigens than S-antigen alone are potential autoimmune targets in TR. These findings parallel observations in onchocerciasis where autoantibody reactivity to retinal antigen distinct from S-antigen or IRBP has been detected in the serum and ocular fluids of patients with chorioretinitis. ${ }^{22}$ Further study indicates that the anti-S-antigen and anti-IRBP antibodies that have been demonstrated in onchocerciasis are not specific to the disease. ${ }^{23}$ Immunological cross reactivity between an onchocercal antigen (Ov39) and a human retinal antigen (hr44) has been confirmed ${ }^{24} 25$ and it has been suggested that antiretinal antibodies may have a role in the development of ocular pathology. 22

Toxoplasma retinochoroiditis is a complex disease; little is known about parasite entry into the retina, the precise location of the parasite once entry is made, and, crucially, the factors that determine disease recurrence. One possible trigger is postinfective autoimmunity. Here, infectious agents may predispose to, or precipitate, autoimmune reactions by unmasking sequestered potential autoantigens by local hypersensitivity reactions. These newly exposed autoantigens may provide the stimulus for the generation of autoreactivity. Alternatively, infectious agents may provoke cross reactive autoimmunity (as in the onchocerciasis model). The demonstration that such mechanisms occur either singly or collectively in TR would strengthen the rationale for postinfective autoimmunity in the pathogenesis of the disease. One supporting factor for the autoimmune hypothesis is the consistent finding of selective photoreceptor loss in animal models of toxoplasma retinochoroiditis. $^{26-29}$ This preferential destruction is particularly interesting when one considers that the location of the encysted parasite is in the neural retina. ${ }^{27}$ The sparing of the neural layers from immune mediated damage in these studies is also reminiscent of the histological appearance of experimental autoimmune uveitis (EAU) induced by administration of 
uveitogenic retinal antigens suggesting a common mechanism of tissue destruction.

A spectrum of possible roles for human antiretinal autoantibodies in the pathogenesis of toxoplasma retinochoroiditis may be considered. A primary role for a humoral autoimmune response in the pathogenesis of TR seems unlikely as antiphotoreceptor antibodies were also found in a number of normal controls, were absent in a small subset of TR patients, and there was a lack of correlation of antiretinal antibody titre with TR disease activity. Conversely, antiretinal antibodies in TR do not appear to be an epiphenomenon of retinal damage as the incidence of these antibodies in IRV patients was significantly lower than that in TR. The hypothesis we favour is that the autoantibodies are copathogenic with cellular responses in TR. In this hypothesis we suggest the antiretinal antibodies are able to reach the retina via the local vasculature, with local concentrations increased at times of compromise of the bloodretinal barrier such as toxoplasma induced inflammation, and thus contribute to retinal damage. Antiphotoreceptor antibody binding may activate the classic pathway of complement with subsequent photoreceptor cell lysis. The binding of antiphotoreceptor antibody to the rod inner segments may also facilitate photoreceptor damage by effector cells. Such an illustration of effector cell mediated photoreceptor loss is recorded in one of the murine models of TR where macrophages have been observed histologically to disrupt non-infected photoreceptor cells, presumably by (attempted) phagocytosis or antibody dependent cellular cytotoxicity. ${ }^{27}$

Regardless of the actual mechanism involved in TR pathogenesis our study provides strong preliminary evidence of antiretinal antibodies in TR against human tissue. Further investigations are now required to determine the antigenic specificity, characteristics, and role of the antiphotoreceptor autoantibodies in the pathogenesis of toxoplasma retinochoroiditis.

The authors would like to thank Dr L Donoso, Wills Eye Hospital, Philadelphia, USA for the supply of antiretinal S-antigen monoclonal antibody and the Bristol Eye Bank, UK for donor eyes. We are grateful to the Toxoplasma Reference Laboratory, St George's Hospital Medical School for performing the toxoplasma serology.

This study was supported by grants from the Iris Fund for the Prevention of Blindness.

1 Kean B. Clinical toxoplasmosis- 50 years. Trans $R$ Soc Trop Med Hyg 1972;66:549-571.

2 Glasner P, Silveira C, Kruszon-Moran D, et al. An unusually high prevalence of ocular toxoplasmosis in southern Brazil. Am f Ophthalmol 1992;114:136-44.
3 Gilbert R, Stanford MR, Jackson $\mathrm{H}$, et al. Incidence of acute symptomatic toxoplasma retinochoroiditis in south London according to country of birth. BMf 1995;310:103740.

4 Perkins E. Ocular toxoplasmosis. Br F Ophthalmol 1973;57: $1-17$

5 Friedmann CT, Knox DL. Variations in recurrent active toxoplasmic retinochoroiditis. Arch Ophthalmol 1969;81: $481-93$.

6 Ho-Yen D. Clinical features. In: Ho-Yen D, Joss A, eds. Human toxoplasmosis. 2nd ed. Oxford: Oxford Medical Publications, 1992:56-78.

7 Dutton G. The causes of tissue damage in toxoplasma retinochoroiditis. Trans Ophthalmol Soc UK 1986;105:404-

8 Rao N, Font R. Toxoplasmic retinochoroiditis; electronmicroscopic and immunofluorescence studies in formalinfixed tissue. Arch Ophthalmol 1977;95:273-7.

9 Yeo J, Jakobiec F, Iwamoto T, et al. Opportunistic toxoplasmic retinochoroiditis following chemotherapy for systemic lymphoma: a light and electron microscopic study. Ophthalmology 1983;90:885-98.

10 Frenkel J. Pathogenesis of toxoplasmosis with a consideration of cyst rupture in besnoitia infection. Surv Ophthalmol 1961;6:799-825.

11 Frenkel J. Ocular toxoplasmosis. Pathogenesis, diagnosis and treatment. Arch Ophthalmol 1986;59:260-79.

12 Hogan M, Moschini G, Zardi O. Effects of Toxoplasma gondii toxin on the rabbit eye. Am $f$ Ophthalmol 971;72:733-42.

13 Rothova A. Ocular involvement in toxoplasmosis. $\mathrm{Br} f \mathrm{Oph}$ thalmol 1993;77:371-7.

14 Newman PE, Ghosheh R, Tabbara KF, et al. The role of hypersensitivity reactions to toxoplasma antigens in experimental ocular toxoplasmosis in non-human primates. $A m \mathcal{F}$ Ophthalmol 1982;94:159-64.

15 Nussenblatt R, Gery I, Ballintine E, et al. Cellular immune responsiveness of uveitis patients to retinal S-antigen. $A m \mathcal{F}$ Ophthalmol 1980;89:173-7.

16 Wyler D, Blackman H, Lunde M. Cellular hypersensitivity to toxoplasmal and retinal antigens in patients with toxoplasma retinochoroiditis. Am f Trop Med Hyg 1980;29: $1181-6$

17 Gregerson D, Abrahams I, Thirkill C. Serum antibody levels of uveitis patients to bovine retinal antigens. Invest Ophthalmol Vis Science 1981;21:669-672.

18 Abrahams I, Gregerson D. Longitudinal study of serum antibody responses to retinal antigens in acute ocular toxoplasmosis. Am f Ophthalmol 1982;93:224-31.

19 Kijlstra A, Hoekzema, Van der Lelij A, et al. Humoral and cellular reactions against retinal antigens in clinical disease. Curr Eye Res 1990;9 (suppl):85-9.

20 Dua HS, Gomes JA, Singh A, et al. Fresh-frozen cucumber as a mount for conjunctival and corneal tissue in cryomicrotomy. Arch Ophthalmol 1994;112:1139-41.

21 Kasp E, Banga JP, Brown EC, et al. An improved method for the purification of retinal S-antigen using selective hydrophobic absorption chromotography. F Immunol Methods 1987; 100:147-52.

22 Chan C, Nussenblatt RB, Kim MK, et al. Immunopathology of ocular onchocerciasis. 2. Anti-retinal autoantibodies in serum and ocular fluids. Ophthalmology 1987;94:439-43.

23 Van der Lelij, Doekes G, Hwan BS, et al. Humoral autoimmune response against S-antigen and IRBP in ocular onchocerciasis. Invest Ophthalmol Vis Sci 1990;31:1374-80

24 Braun G, McKechnie NM, Connor V, et al. Immunological cross-reactivity between a cloned antigen of Onchocerca volvulus and a component of the retinal pigment epithelium. F Exp Med 1991;174:169-77.

25 McKechnie NM, Braun G, Klager S, et al. Immunological cross-reactivity in the pathogenesis of ocular onchocerciasis. Invest Ophthalmol Vis Sci 1993;34:2888-902.

26 Dutton G, Hay J. Toxoplasmic retinochoroiditis-current concepts in pathogenesis. Trans Ophthalmol Soc UK 1983;103:503-7.

27 Dutton G, McMenamin P, Hay J, et al. The ultrastructural pathology of congenital murine toxoplasmic chorioretinitis; II. The morphology of the inflammatory changes. Exp Eye Res 1986;43:545-60

28 Yoshizumi M. Experimential Toxoplasma retinitis. A light and electron microscopical study. Arch Pathol Lab Med 1976;100:487-90.

29 Ashton N. Ocular toxoplasmosis in wallabies (Macropus rufogriseus). Am f Ophthalmol 1979;88:322-32. 